\title{
Uma nova espécie do gênero Temnomastax (Temnomastacinae, Eumastacidae, Orthoptera) da Amazônia
}

\author{
Renan S. Olivier
}

Programa de Pós-Graduação em Biologia Animal, Laboratório de Zoologia, Centro de Ciências Biológicas e da Saúde, Universidade Federal de Mato Grosso do Sul (UFMS), 79090-900, Campo Grande, MS, Brasil. (renanolivier91@gmail.com)

\begin{abstract}
A new species of genus Temnomastax (Temnomastacinae, Eumastacidae, Orthoptera) of Amazon. The Temnomastax genus currently has seven species described, widely distributed at the Cerrado domain in South America. In this work an eighth species, Temnomastax spielmanni sp. nov., is described from the Brazilian Amazon region. The diagnosis of this new species is based on the phallic complex characters and external morphological characteristics. Temnomastax spielmanni sp. nov. resembles Temnomastax beni Rehn \& Rehn, 1942, occurring in Bolivia, but can be differentiated by some external characters. The phallic complex of $T$. spielmanni sp. nov. presents characters not yet known in Temnomastacinae; this fact reinforces the need for a revision of this subfamily, which may clarify the existing phylogenetic relationships.
\end{abstract}

KEYWORDS. Phallic complex, external morphology, distribution.

RESUMO. O gênero Temnomastax apresenta atualmente sete espécies, sendo amplamente distribuídas pelo domínio Cerrado, na América do Sul. Neste trabalho, uma oitava espécie, Temnomastax spielmanni sp. nov., é descrita para a região Amazônica brasileira. A diagnose da nova espécie baseia-se em caracteres do complexo fálico e em características morfológicas externas. Temnomastax spielmanni sp. nov. apresenta semelhança com Temnomastax beni Rehn \& Rehn, 1942, que ocorre em território boliviano, mas pode ser diferenciada por alguns caracteres externos. O complexo fálico de T. spielmanni sp. nov. apresenta caracteres ainda não descritos em Temnomastacinae; tal fato reforça a necessidade da revisão taxonômica dessa subfamília, o que poderá esclarecer as relações filogenéticas existentes.

PALAVRAS-CHAVE. Complexo fálico, morfologia externa, distribuição.

Temnomastax Rehn \& Rehn, 1942 apresenta atualmente sete espécies descritas: T. borellii (GiglioTos, 1897), T. tigris (Burr, 1899), T. beni Rehn \& Rehn, 1942, T. chiquitos Rehn \& Rehn, T. hamus Rehn \& Rehn, T. latens Rehn \& Rehn, 1942 e T. ricardoi Descamps, 1973 (EADEs et al., 2014). Os caracteres da morfologia externa mais evidentes em tal gênero são o fastígio truncado, asas com variados graus de desenvolvimento e tergitos 7 e 8 com coloração diferenciada. Quanto ao complexo fálico, o epifalo é discoidal, com contornos esclerotizados e centralmente membranoso, o ectofalo é membranoso e o esclerito endofálico possui formato de U (Rehn \& Grant JR., 1958; Descamps, 1973a; Eades et al., 2014). Tais características da genitália masculina são importantes também para diferenciação entre os dois gêneros da subfamília Temnomastacinae, já que as espécies de Eutemnomastax Descamps, 1979 apresentam variações no formato do epifalo e o esclerito endofálico é curvado em vista lateral.

Temnomastax é distribuído por todo o planalto brasileiro (Descamps, 1973a; EAdes et al., 2014). Atualmente existem registros nos estados de Minas Gerais, São Paulo, Goiás, Mato Grosso e Mato Grosso do Sul, além de outras ocorrências em países limítrofes como Argentina, Paraguai e Bolívia, indicando, portanto, uma distribuição por todo o domínio Cerrado em todo o centro da América do Sul. Apenas T. beni apresenta distribuição fora desse padrão, pois é registrada até o momento apenas ao norte da Bolívia, no município de Rurrenabaque, região que pertence à Amazônia boliviana. Objetiva-se descrever uma nova espécie do gênero Temnomastax, a primeira registrada na região do domínio Amazônico em território brasileiro.

\section{MATERIAL E MÉTODOS}

O único espécime foi coletado no município de Guajará-Mirim no estado de Rondônia, Brasil. Está conservado em via seca e pertence à coleção entomológica do Instituto Nacional de Pesquisas da Amazônia (INPA, Manaus/AM).

Para a descrição do espécime foram utilizados como base Rehn \& Grant JR. (1958), Descamps (1973a) e Rowell \& Bentos-Pereira (2001). As medidas foram baseadas em Carbonell (2002).

Para descrever caracteres morfológicos externos e do complexo fálico o espécime foi observado sob estereomicroscópio Leica DFC420. Para o estudo do complexo fálico, o espécime foi dissecado utilizando-se a técnica de Dirsh (1956), que consiste em imersão da estrutura em solução de $\mathrm{KOH}(10 \%)$ até que o tecido muscular esteja totalmente digerido; posteriormente a peça foi lavada com água destilada e, ao final, foi armazenado em glicerina. Para aferir as medidas do espécime utilizouse o software Leica Application Suite V3.8. 
Temnomastax spielmanni sp. nov.

(Figs 1-4)

Material-tipo. Holótipo $\widehat{\text { }}$, BRASIL, Rondônia, Guarajá-Mirim, 07-11.IX.1999 (INPA, Manaus/AM).

Diagnose. Pequeno porte $(1,404 \mathrm{~cm})$, delgado e macróptero; fastígio em vista dorsal com bordas laterais salientes; cercos cônicos curvados para região mediana do epiprocto em vista dorsal; epifalo posteriormente arredondado e com região escurecida trilobada em visão dorsal; endofalo curvado em vista lateral; ectofalo posteriormente esclerotizado e com dois pares de espinhos em sua extremidade distal.

Descrição. Cabeça. Antena com 12 antenômeros, escapo e pedicelo amarelo, primeiro antenômero do flagelo amarelo/preto e os demais pretos. Órgão antenal não evidente. Fastígio evidente em vista lateral e bordas laterais salientes em vista dorsal. Olhos globosos e elípticos. Fronte amarela, clípeo amarelo com máculas azuis, labro azul, palpos e mandíbulas amarelas. Articulações mandibulares enegrecidas. Região lateral da cabeça preta, com uma banda transversal clara na região entre olho e gena. Região occipital escura com marcas claras (cor semelhante à banda transversal anteriormente citada). Membrana cervical amarela no dorso e marrom lateralmente.

Tórax. Pronoto enselado. Disco com borda posterior levemente arredondada em vista dorsal. Ampla faixa amarela no disco em vista dorsal. Lobo lateral do pronoto com ângulo ínfero-anterior arredondado e ínfero-posterior agudo e pouco projetado posteriormente. Lobo lateral do pronoto com gradiente do marrom para o amarelo, da região superior para a inferior. Tergitos torácicos com porção superior amarela e inferior enegrecida. Asas: Macróptero. Tégminas e asas ultrapassam em aproximadamente 1/3 o final do abdome, ápice arredondado, membranas amareladas e translúcidas e venação marrom. Pernas: anteriores e médias amarelas, com porção distal da protíbia, mesotíbia e tarsos de coloração azul esverdeada. Carenas dorsais do profêmur pouco evidentes. Pro- e mesotíbia com nove espinhos ventrais internos e nove ventrais externos. Metafêmur amarelo escurecido com um anel mais claro no terço distal, apicalmente marrom. Metatíbia marrom-clara, com 26 espinhos na região dorsal externa e 27 internos, distalmente com três esporões, dois internos e um externo. Espinho dorso-externo no primeiro metatarsômero. Garras tarsais de comprimento desigual e serreadas internamente.

Abdome. Tergitos amarelos com borda posterior marrom. Tergitos 7 e 8 mais escuros que os demais. Placa supra-anal alargada na região proximal e trigonal distalmente, presença de área membranosa na região central. Esternitos amarelos com algumas manchas dispersas marrons. Placa subgenital com ampla área membranosa, margem posterior esclerotizada, região mediana dessa não projetada posteriormente. Cercos cônicos curvados para região mediana do epiprocto em vista dorsal.
Complexo fálico (Figs 2-4). Epifalo: Membranoso, com porções laterais e posterior esclerotizadas (Fig. 2), porções laterais não se ligam anteriormente e formam ampla área membranosa em forma de $\mathrm{U}$ ao centro. Projeções laterais curvadas ventralmente e estendidas posteriormente, afiladas distalmente. Porção posterior arredondada e com uma região escurecida trilobada (Fig. 2). Epifalo convexo em vista lateral, com porção posterior côncava (Fig. 4). Endofalo (Fig. 4) curvado em vista lateral, com extremidades posteriores dos braços divergindo lateralmente. Ectofalo: membranoso em sua maior extensão, com ápices da extremidade posterior esclerotizados (Figs. 2-4), havendo em cada uma das duas porções dois processos em forma de espinho (Fig. 2). Em vista axial, os espinhos em posição superior são mais distantes entre si, curvados distalmente e levemente inclinados ventralmente, os inferiores mais próximos entre si e curvados distalmente, tal conformação entre as duas porções do ectofalo acoplam dorsalmente a porção posterior do epifalo (Fig. 3).

Mensurações (mm). Comprimento do fastígio ao final da tégmina 14,04; comprimento do fastígio ao final do abdome 11,27; comprimento do fastígio 0,12 ; comprimento do pronoto (p) 1,46; comprimento da cabeça + pronoto 3,52; largura da cabeça gena-gena 1,49; comprimento do metafêmur (mf) 8,40; largura do metafêmur 1,30; comprimento da tégmina (t) 10,38; maior diâmetro do olho 1,22; menor diâmetro do olho 0,86; distância interocular 0,31. Razões: t/mf 1,23; t/p 7,10; mf/p 5,75

Distribuição. Espécie conhecida apenas para a localidade-tipo.

Etimologia. Epíteto específico em homenagem ao Dr. Adriano Afonso Spielmann da Universidade Federal de Mato Grosso do Sul, exímio professor, liquenólogo e pessoa pela qual tenho grande admiração.

\section{Chave de identificação para machos adultos de Temnomastax Rehn \& Rehn, 1942}

1. Indivíduo macróptero .2

1'. Indivíduo braquíptero .3

2(1). Primeiro metatarsômero com espinho dorsal externo. Placa subgenital sem projeção mediana na sua borda posterior. Cercos com curvatura acentuada (Fig. 7) .............

Temnomastax spielmanni sp. nov.

2'. Primeiro metatarsômero sem espinho dorsal externo. Placa subgenital com projeção mediana na sua borda posterior. Cercos com curvatura suave (Fig. 5)

Temnomastax beni Rehn \& Rehn, 1942

3(1'). Cercos comprimidos lateralmente (Figs 8-10) ........ (grupo tigris) 4

3’. Cercos não comprimidos lateralmente (Figs 5-7, 11, 12)

4(3). Curvatura da borda superior dos cercos tênue e ângulos ínfero-posteriores com processo odontiforme (Fig. 8) Temnomastax borellii (Giglio-Tos, 1897)

4'. Curvatura da borda superior dos cercos de outra forma 


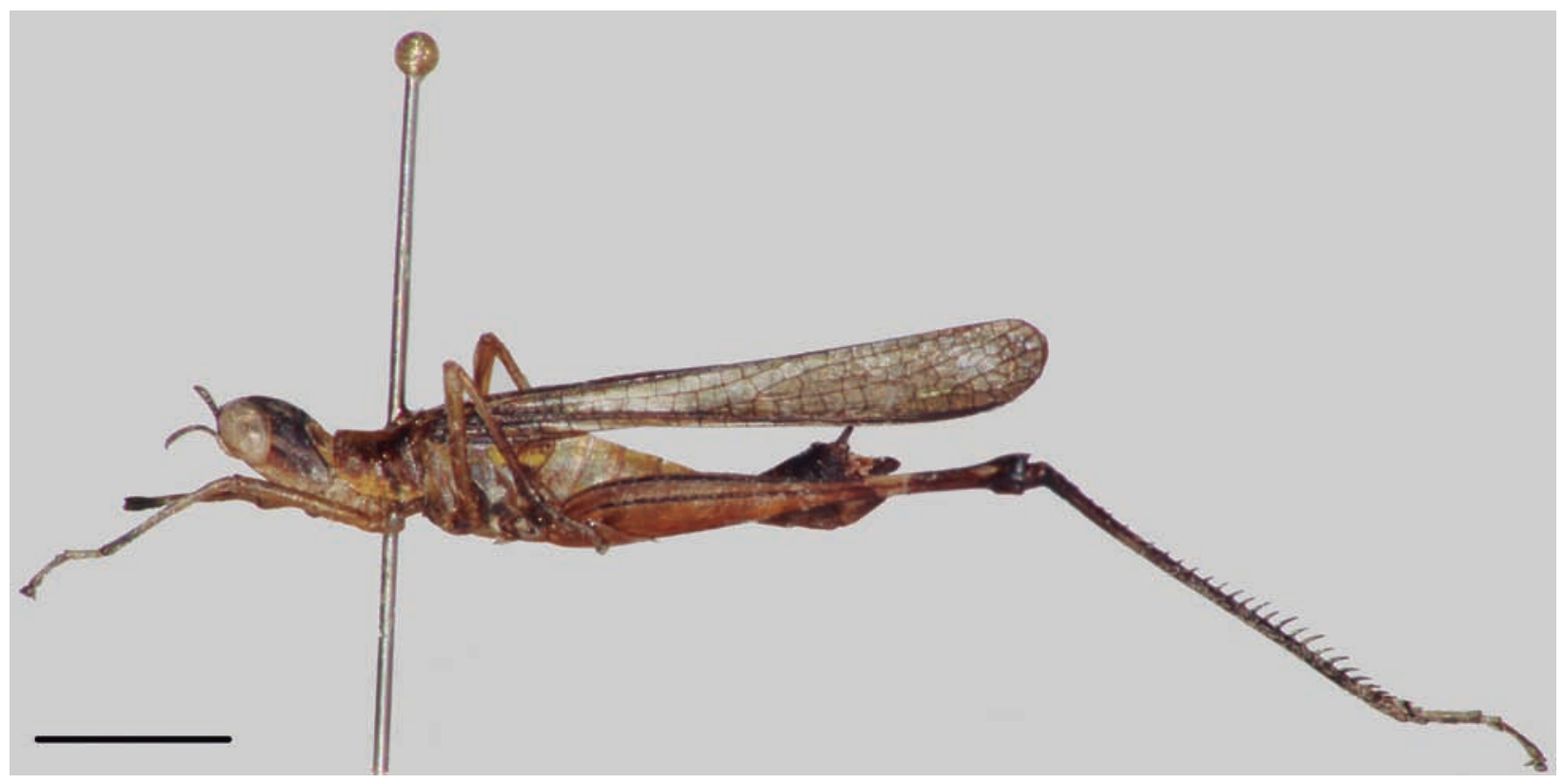

Fig. 1. Temnomastax spielmanni sp. nov., holótipo ổ, hábito, vista lateral. Escala, $3 \mathrm{~mm}$.

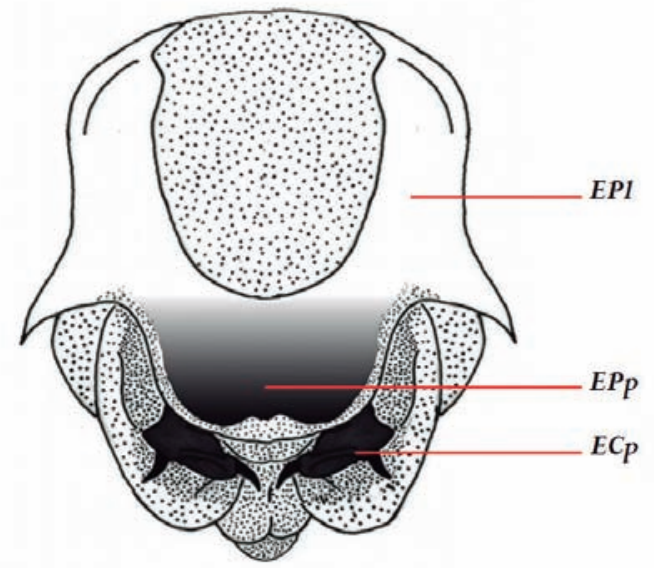

2

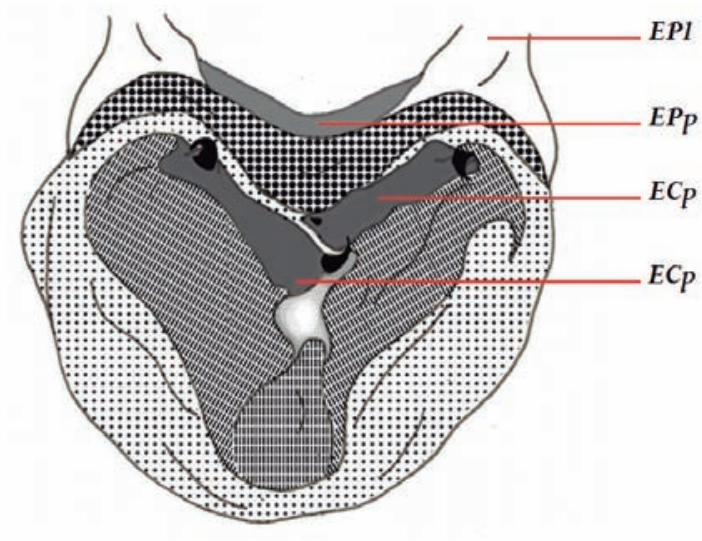

3

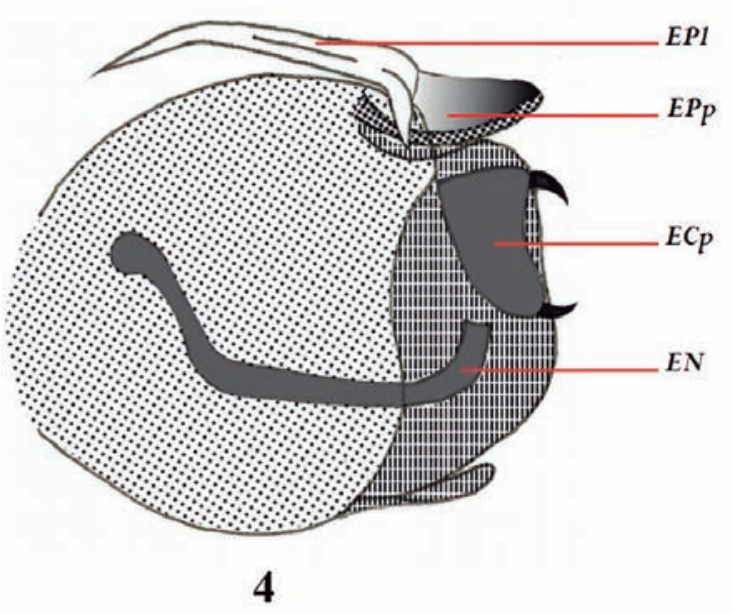

Figs 2-4. Temnomastax spielmanni sp. nov., complexo fálico: 2, vista dorsal; 3, vista axial; 4, vista lateral (EPl, porções laterais do epifalo; $E P p$, porção posterior do epifalo; EN, Endofalo; ECp, porção posterior do ectofalo). Escala, 0,5 mm. 


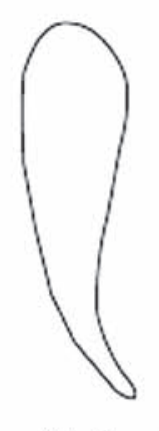

T. beni 5

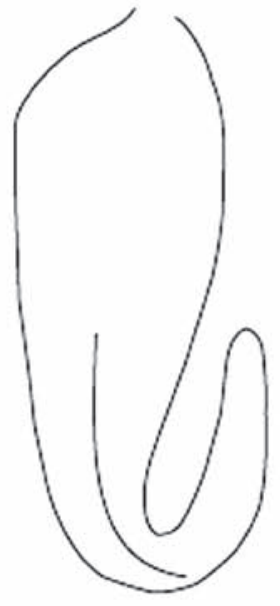

T. hamus 11

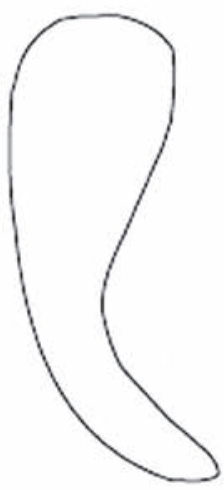

T. latens

6

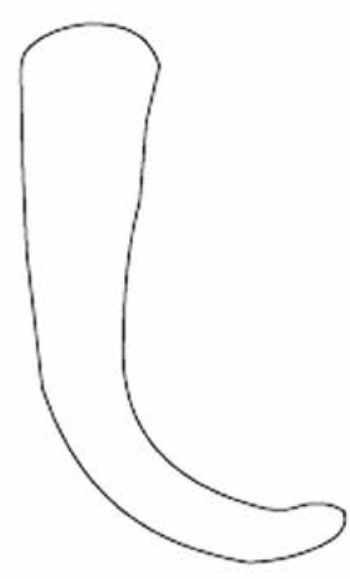

T. spielmanni

7

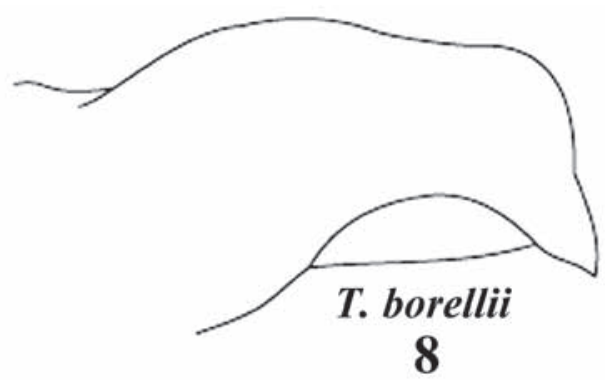

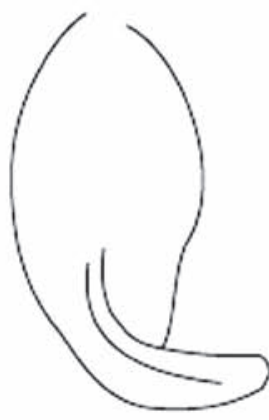

T. chiquitos 12

Figs 5-12. Cercos masculinos de espécies de Temnomastax Rehn \& Rehn, 1942: 5-7, 11,12, vista dorsal; 8-10, vista lateral. Figs 5, 6, 8-12 foram retirados de Descamps (1973b).

e ângulos ínfero-posteriores sem processo odontiforme 5

5(4’). Tégminas e asas longas, atingindo a borda posterior do $4^{\circ}$ tergito. Cercos com borda superior levemente curvada, ângulos posteriores mais curvados internamente (Fig. 9) Temnomastax tigris (Burr, 1899)

5'. Tégminas e asas curtas, não atingindo a borda posterior do $4^{\circ}$ tergito. Cercos com borda superior acentuadamente curvada, ângulos posteriores menos curvados internamente (Fig. 10) ......... Temnomastax ricardoi Descamps, 1973 6(3'). Cercos com curvatura uniforme (Fig. 6). Tégminas e asas atingindo o ápice do abdome

Temnomastax latens Rehn \& Rehn, 1942

6'. Cercos com curvatura acentuada distalmente; tégminas e asas não atingindo o ápice do abdome

7(6'). Cercos longos acentuadamente curvados, em forma de gancho (Fig. 11) ....

Temnomastax hamus Rehn \& Rehn, 1942

7’. Cercos curtos e posteriormente levemente curvados

(Fig. 12) .Temnomastax chiquitos Rehn \& Rehn, 1942.

\section{DISCUSSÃO}

A espécie mais semelhante a T. spielmanni sp. nov. é T. beni, em características como: macropterismo, conformação do pronoto e os cercos cônicos ligeiramente curvados, sendo estas evidentes em uma observação preliminar. Entretanto, alguns caracteres de T. spielmanni sp. nov. distinguem de $T$. beni: fastígio com bordas laterais salientes dorsalmente, placa subgenital com região mediana posterior não projetada e espinho dorsal externo no primeiro metatarsômero. Complementarmente às semelhanças morfológicas entre essas duas espécies, a proximidade geográfica sugere uma relação filogenética entre ambas. Temnomastax spielmann sp. nov. expande a distribuição geográfica de Temnomastax para a região Amazônica brasileira. Com a defasagem do conhecimento de Temnomastacinae e, adicionalmente, o reduzido número de espécimes conhecidos dessas duas espécies (apenas exemplares-tipos) a determinação em nível genérico é limitada. Entretanto, com base no atual conhecimento do grupo, a nova espécie integra o gênero Temnomastax. 
Algumas características são distintas e divergem do observado por Rehn \& Grant JR. (1958) para os Temnomastacinae, em especial para Temnomastax. O ectofalo com processo especializado e a curvatura do endofalo em T. spielmanni sp. nov. são semelhantes ao encontrado em Eumastacops nemorivaga Rehn \& Rehn, 1942, que pertence a uma subfamília próxima, Eumastacopinae. O mesmo ocorre com o fastígio que, em vista dorsal, apresenta duas carenas laterais, característica dessa mesma subfamília. Em contrapartida, a nova espécie possui espinho no primeiro metatarsômero, além da esclerificação epifálica e ectofálica não ser complexa, o que, segundo Descamps (1979), não permite a sua classificação nos gêneros Eumastacops Rehn \& Rehn, 1942 e Pseudeumastacops Descamps, 1974, respectivamente. De modo complementar Descamps (1979), ao descrever Eutemnomastax, afirma que $T$. beni detém algumas características peculiares, as quais não são encontradas comumente em Temnomastax e Eutemnomastax.

Até o presente momento, o complexo fálico de $T$. beni não foi descrito, limitando portanto uma maior clareza da relação entre essas duas espécies. É importante salientar que um estudo mais aprofundado de Temnomastacinae se faz necessário, em especial com essas duas espécies tão peculiares que ocorrem na Região Amazônica, e com isso, possivelmente a classificação genérica será esclarecida.

Agradecimentos. A Cristiane Vieira de Assis Pujol (UCB), Gustavo Graciolli (UFMS), Oscar J. Cadena Castañeda (Universidade Distrital Francisco José de Caldas, Colômbia) e aos dois revisores anônimos por críticas e sugestões ao manuscrito. A Andressa F. de Oliveira (UFMS) pela elaboração da Fig. 1 e Nathalia Borine pela edição das Figs 2-4. Ao INPA, Manaus, AM, em especial a Marcio Luiz de Oliveira, por fornecer o espécime para estudo.

\section{REFERÊNCIAS BIBLIOGRÁFICAS}

CARBonell, C. S. 2002. The grasshopper tribe Phaeopariini (Acridoidea: Romaleidae). Philadelphia, The Orthopterists' Society. 148p.

Descamps, M. 1973a. Révision des Eumastacoidea (Orthoptera) aux échelons des familles et des sous familles (genitalia, répartition, phylogénie). Acrida 2:161-298.

. 1973b. Diagnoses et signalisations d'Eumastacoidea (Ortho.) IV. Amerique. Annales de la Société Entomologique de France 9(4):943-974.

. 1979. Eumastacoidea néotropicaux diagnoses, signalisations, notes biologiques. Annales de la Société Entomologique de France 15(1):117-155.

Dirsh, V. M. 1956. The phallic complex in Acridoidea in relation to taxonomy. Transactions of the Royal Entomological Society of London 108(7):223-270.

Eades, D. C.; Otte, D.; Cigliano, M. M. \& Braun, H. 2014. Orthoptera Species File Online, Version 5.0/5.0. Disponível em: <http:// Orthoptera.SpeciesFile.org>. Acesso em: 02.01.2014.

Rehn, J. A. G. \& Grant, JR. H. J. 1958. The phallic complex in the subfamilies of New World Eumastacidae and the family Tanaoceridae. Proceedings of the Academy of Natural Sciences of Philadelphia 110:301-319.

Rowell, C. H. F. \& Bentos-Pereira, A. 2001. Review of the genus Homeomastax (Eumastacinae, Eumastacidae, Eumastacoidea, Orthoptera), with description of new species. Journal of Orthoptera Research 10(2):209-254. 